\title{
Article
}

\section{Development of a Method to Identify in- Plane Anisotropy Axes in Soft Magnetic Materials Using a Standard Vibrating Sample Magnetometer}

Bourn, Steven, Mercer, Tim, Bissell, Phil and Vopson, Melvin

Available at http://clok.uclan.ac.uk/12740/

Bourn, Steven, Mercer, Tim ORCID: 0000-0002-1557-2138, Bissell, Phil and Vopson, Melvin (2015) Development of a Method to Identify in-Plane Anisotropy Axes in Soft Magnetic Materials Using a Standard Vibrating Sample Magnetometer. IEEE Transactions on Magnetics, 51 (11). ISSN 0018-9464

It is advisable to refer to the publisher's version if you intend to cite from the work. http://dx.doi.org/10.1109/TMAG.2015.2435365

For more information about UCLan's research in this area go to http://www.uclan.ac.uk/researchgroups/ and search for <name of research Group>.

For information about Research generally at UCLan please go to http://www.uclan.ac.uk/research/

All outputs in CLoK are protected by Intellectual Property Rights law, including Copyright law. Copyright, IPR and Moral Rights for the works on this site are retained by the individual authors and/or other copyright owners. Terms and conditions for use of this material are defined in the policies page. 


\title{
Development of a Method to Identify In-Plane Anisotropy Axes in Soft Magnetic Materials Using a Standard Vibrating Sample Magnetometer
}

\author{
Steven Bourn ${ }^{1}$, Tim Mercer ${ }^{1,3}$, Phil Bissell ${ }^{1}$, and Melvin Vopson ${ }^{2}$ \\ ${ }^{1}$ Jeremiah Horrocks Institute for Mathematics, Physics and Astrophysics, University of Central Lancashire, Preston PR1 2HE., \\ United Kingdom \\ ${ }^{2}$ Faculty of Science, University of Portsmouth, Portsmouth PO1 3QL., United Kingdom \\ ${ }^{3}$ Department of Physics, University of Liverpool, Liverpool, L69 7ZE, U.K.
}

A method of identifying in-plane anisotropy axes in soft magnetic materials has been developed using an in-field-only measurement technique. The method is based on an extended bi-axial Vibrating Sample Magnetometer (VSM) technique that simulates the torque on a sample; giving rise to equivalent torque curves that are comparable with those determined directly using a torque magnetometer. Testing of the new methodology was carried out on magnetically soft and multi-axial nickel ferrite textured films deposited with various crystal orientations. The results compare well with the accepted bi-axial VSM technique, identifying the same in-plane anisotropy directions and relative easy and hard axes from the in-field measurement alone. This means that these characteristics could be determined using a standard VSM measuring magnetization in the field direction as long as it is fitted with a rotating sample stage.

Index Terms-In-Plane Anisotropy Axes Directions, Soft Magnetic Materials. Torque Magnetometry, Vibrating Sample Magnetometry, Angular Dependence, Easy and Hard Axes

\section{INTRODUCTION}

$\mathrm{K}_{\mathrm{n}}^{\mathrm{N}}$ NOWLEDGE OF the anisotropic behavior of magnetic materials is important in the characterization of soft and hard materials and development of an understanding of their characteristics. For example, Garcia et al [1] have studied the relation of anisotropy to magnetostriction in nanocrystalline melt spun Fe-Al alloys before and after annealing. Provided the direction of hard axes is known Dubuget et al [2], furthering work by Barandiaran et al [3], have shown a method of characterizing the anisotropy field distribution by measurements along a sample's hard axis.

Torque magnetometry, which directly measures the couple acting on a sample generated by the magnetic field, is the traditional method of determining magnetic anisotropic axes and the analysis is well documented in books and other literature [e.g. 4] and is still in use [e.g. 5]. An alternative is to simulate the torque on a sample using the in-field and perpendicular magnetization as a sample is rotated and measured using a bi-axial Vibrating Sample Magnetometer (VSM). Although these magnetometers are now available in many laboratories, they are not universally available and many studies are still restricted to the in-field direction.

Torque measurements are especially useful in characterizing the in-plane 'easy' and 'hard' axes in soft magnetic samples. We have been using a bi-axial VSM to simulate torque measurements and to identify the 'easy' and 'hard' axes of multi-axial textured magnetically soft nickel ferrite $\mathrm{NiFe}_{2} \mathrm{O}_{4}$ films deposited with various crystal orientations, and by measuring the in-field and transverse magnetization during rotation of the sample.

During these studies, we have identified a relation between the in-field and the transverse magnetization involving a phase shift which indicates that it is possible to determine the direction and type of in-plane anisotropy axes in these

\footnotetext{
Manuscript received January 1, 2008 (date on which paper was submitted for review). Corresponding author: F. A. Author (e-mail: f.author@nist.gov).

Digital Object Identifier inserted by IEEE
}

materials from the in-field magnetization alone. This means that these characteristics could be determined using a standard VSM (measuring magnetization in the field direction) providing a rotating sample stage is fitted to it. Whilst other workers [6], [7] have developed rotational in-field methods, these are based on the theory of uniaxial anisotropy energy and require fitting of the theory to their experimental data in order to determine parameters that include axis type (easy or hard) and direction in their uniaxial samples. In contrast, our proposed method is not limited to uniaxial samples and only requires a simple and direct data analysis to obtain axis type and direction on fully soft films that have closed magnetization loops along their easy axes.

In this paper we describe our bi-axial VSM measurements on soft nickel ferrite samples and show the relation between in-field and transverse measurements and how the anisotropy axes types and directions can be determined from the in-field measurement alone. Whilst we will continue to use bi-axial measurements, this analysis may be useful to researchers who only have in-field measurements available in their laboratories.

\section{EXPERIMENTAL METHODOLOGY}

\section{A. Samples and Experimental Setup}

Thick $\mathrm{NiFe}_{2} \mathrm{O}_{4}$ (NFO) polycrystalline films of $\sim 1.25 \mathrm{~mm}$ and with known crystallographic orientation [8] were used in this study. The films had been textured along a favored direction so that a sample annotated as say NFO_100 refers to orientation parallel to a plane of the form $\{100\}$. The samples had been fabricated as discs of diameter $\sim 8 \mathrm{~mm}$ to ensure uniformity of shape (with respect to the applied field and pickup coils of the VSM) as a function of angular rotation in the measurement plane

Magnetic characterization was carried out using a UCLan built in-house $10 \mathrm{kOe}$ bi-axial VSM. It includes precision control of the sample position that is used to effectively 
eliminate any precession that could otherwise occur during sample rotation.

\section{FIG. 1 HERE}

\section{B. Extending the Conventional Bi-Axial VSM Methodology in order to lessen the Effects of Saturation}

In general, by consideration of the torque per unit volume, $\boldsymbol{L}=\boldsymbol{M} \times \boldsymbol{H}$, experienced by a sample of magnetization, $\boldsymbol{M}$, in an applied field, $\boldsymbol{H}$, then a bi-axial VSM provides a convenient method for determination of the couple that would otherwise act on the sample in the conventional direct measurement technique. From the bi-axial geometry of the pick-up coils, with one set parallel to the in-field direction and the other transverse in the same measurement plane, then the component of $\boldsymbol{M}$ in-field will generate zero torque and the general equation reduces to

$L=M_{\perp} H$,

where $M_{\perp}$ is the perpendicular magnetization obtained from the transverse coil set. Plotting this equivalent torque as a function of sample rotation simulates the torque curves obtained by direct measurement and is thereby an accepted method for the determination of anisotropy axes in magnetic materials.

A known limitation of the bi-axial method is that when maximum torque is achieved as a sample approaches saturation, then $M_{\perp}$ is being reduced to a minimum (and tending to zero) due to nearly all of the vector sample moment, $\boldsymbol{m}$, being pulled into the applied field direction. In our study on soft samples with saturation fields of about 1.5 kOe we have been routinely using a modified methodology, whereby a series of simulated rotational torque curves have been generated at decreasing field steps. In each case, the sample is saturated whilst static and the field then reduced to a value lower than the previous step before rotation takes place. In this manner, a distinctive repeat pattern is observed as shown in Fig. 1 for the NFO_100 sample.

Here the sample was initially saturated at $10 \mathrm{kOe}$ for each curve prior to rotation in the non-saturating range $0.6 \mathrm{kOe}$ to 0.2 kOe. From this, a series of common intercepts with the abscissa is observed, with the crossing points that have a positive gradient spaced $90^{\circ}$ apart at $68^{\circ}, 158^{\circ}, 248^{\circ}$ and $338^{\circ}$ respectively. Likewise, a set of common crossing points with a negative gradient are also spaced $90^{\circ}$ apart, starting at $23^{\circ}$ and leading to alternate negative and positive gradient intercepts spaced $45^{\circ}$ apart. This is consistent with one set of perpendicular bi-axial anisotropy easy axes orientated at $45^{\circ}$ with respect to a second set of perpendicular bi-axial hard axes. The terms "easy" and "hard" refer to the relative strengths, in respect of either anisotropy energies or anisotropy fields, of these axes compared to one another. In this sense, one of the bi-axial sets can be thought of as being "harder" than the other set, or alternatively that one set is "easier". It should be noted for these soft materials, where the magnetization will tend to zero at small and decreasing fields, that the features in the torque curve flatten out and disappear as the torque also tends to zero. This can be seen in the 0.2 kOe curve of Fig. 1.

\section{FIG. 2 HERE}

The results for the NFO_100 sample of Fig. 1 compare well with other nickel ferrite samples measured by direct torque magnetometry [9] giving the expected easy and hard axes at the negative and positive gradient intercepts respectively and the subsequent bi-axial anisotropy in the $<111>$ easy directions and the $<100>$ hard directions expected for this orientation of a cubic crystal in the plane of the disk [10]. This gives confidence in our extended bi-axial VSM method and leads to the development of a technique for using the in-field magnetization alone.

\section{DeVelopment And Testing of THE Methodology for USING IN-FIELD MAGNETIZATION ONLY}

\section{A. In-Field Methodology Development}

Consider first a saturated sample in a large applied field that is significantly above the switching field distributions in any of the in-plane directions. If the sample is now rotated through a full $360^{\circ}$ the vector moment, $\boldsymbol{m}$, will always be switched along the in-field direction.

If the field is now reduced to some value below the upper limits of a switching field distribution and is rotated to an angle, $\theta$, with respect to the in-field direction, it will have a net vector moment that no longer lies parallel to the applied field. This is because $\boldsymbol{m}$ now contains both switched and unswitched components instead and whilst the switched component will stay "switched" in-field, the un-switched component will rotate with the sample at $\theta$ resulting in a net vector moment at an angle $\phi$ to the parallel direction.

From simple trigonometry and the magnitude of the moment, $m$, we know that the transverse component of the moment measured in the transverse pick-up coil set of the VSM is simply $m_{\perp}=m \sin \phi$ and that the signal from the infield coil set yields the parallel component $m_{/ /}=m \cos \phi$. This suggests it may be possible to derive the transverse moment needed for simulated torque curves from the in-field signal of a standard VSM, as

$$
\frac{d m_{\|}}{d \phi}=-m \sin \phi=-m_{\perp} .
$$

However, this is only applicable if the angle, $\phi$, is known.

If we now consider the effects of the decreasing field method on our mixed system of switched and un-switched components, it is convenient to think of this as a system of anisotropic "particles" with a distribution of switching fields that are aligned along the various anisotropic axes of the sample. Starting from the limit of saturation, when all the particles are switched under rotation into the parallel direction 
of $\theta=$ zero, the reduction of applied field leads to an increase in un-switched particles at the expense of the switched ones. As the un-switched particle moments are not held in the parallel direction they begin to rotate with the sample, becoming ever more closely aligned with the sample rotation angle at each decreasing field step. The combination of the relative decrease in the parallel magnetization component at the same time as the increase in the rotational magnetization component means that the vector moment is now tending to the direction of $\theta$ such that as $\phi \rightarrow \theta$, then (2) becomes valid as a means of simulating torque curves by differentiation of the parallel signal used in standard VSM instruments.

It should be noted that for measurements on soft materials with negligible hysteresis, the technique becomes limited at the small field end due to the drop towards zero of the magnetization signal in these samples at that point.

\section{B. Testing of the Methodology using In-Field Rotation Curves}

The results of applying the methodology to the in-field rotation curves of the NFO_100 sample are shown in Fig. 2. The curves compare well with those of the bi-axial method of Fig. 1, reproducing all the essential features to show two sets of perpendicular bi-axial anisotropy directions $45^{\circ}$ apart.

Whilst these results are encouraging, a better test was provided by using a NFO_110 sample that has a more complex anisotropy. In this case the sample was expected to have three sets of axes, with one set classed as relatively easy, the other medium and the final set hard [10].

The in-field (parallel) curves of magnetization as a function of sample rotation are shown in Fig. 3 for the NFO_110 disk. As such, they can be thought of as the raw data that could be obtained from a standard VSM fitted with an inexpensive rotational stage. Repeating patterns can be observed, such as the two troughs of approximately the same depth at $158^{\circ}$ and $338^{\circ}$. The results of differentiating the curves of Fig. 3 are shown in Fig. 4, where they are displayed as data points to allow comparison with the lines of the bi-axial method that are also shown in the same plot. The two data sets compare well, with the curves crossing the $x$ axis at the same points and with all the finer features of the bi-axial method, such as the relative amplitude of the peaks, apparent using the new methodology.

\section{FIG. 3 HERE}

Again, these crossing points show the positions of the anisotropy axes within the plane of the sample. In addition, inspection of the "standard" VSM data of Fig. 3 provides the means of classifying their relative hardness or easiness. This is due to the different saturating fields that occur at different sample orientations. For example, if the sample is orientated with an easy axis parallel to the applied field it will have a lower saturation field than the same sample with its hard axis also rotated into the field direction. Hence, as our reducing field step methodology takes place below saturation, this ensures that for a given field the easy axis magnetization is at a higher value than that of the harder axis. This is because the easy axis is closer to the saturation point at that field step when compared to that of the harder axis.

\section{FIG. 4 HERE}

Application of this classification methodology to the data of Fig. 3 shows that the highest peaks (marked as E) occur at four crossing points of Fig. 4 at around $34^{\circ}, 98^{\circ}, 210^{\circ}$ and $280^{\circ}$, with just the first one marked "easy" to avoid clutter. It should be noted that the uncertainty in the positions is limited by the $4^{\circ}$ resolution of the rotation steps used.

The first of two medium and two hard axes have also been identified in Fig. 4 from the respective set of intermediate and low moment turning points of Fig. 3 (marked as $\mathrm{M}$ and $\mathrm{H}$ respectively). For a cubic crystal orientated parallel to the $\{110\}$ plane, these axes can be mapped to the $\langle 100\rangle,\langle 110\rangle$ and $<111>$ directions. Closer analysis of the anisotropy axes shows that the hard axes along $<100>$ and the medium axes along $<110>$ are spaced $90^{\circ}$ apart, but that the easy axes at $<111>$ are not at $45^{\circ}$ to either. This is consistent with an imperfect cubic lattice of slightly unequal lattice parameters that can sometimes be a result of strain in the texturing process of film growth.

It should be noted that a lower field-step increment of 40 Oe was needed for the NFO_110 sample of Fig. 4 compared to the 100 Oe increment of the NFO_100 sample of Fig. 2. This was expected due to the more complex anisotropy of the $\{110\}$ plane that requires the vector moment direction to be as close as possible to the sample rotation in order to generate the equivalent transverse moment response of the bi-axial method. Simply reducing the field to lower values to increase the number of un-switched components starts to have a negative effect in soft materials because of the drop-off of the magnetization signal towards zero at low fields. Hence, an optimum or "sweet" point using the new methodology needs to be found between these competing factors. Experimentally this was done by initially using the 100 Oe field increments of the NFO_100 sample over a wide range. This resulted in most of the curves being jagged and with no consistency in the, relative size, shape and number of the peaks and troughs across the applied field range. The next step involved choosing the smoothest grouping of curves in the set that also had the most consistent features. For the NFO_110 sample, this occurred between 500 Oe and 300 Oe, with these values used to define a narrower field range and smaller field-step increments until all the curves were smooth and consistent in terms of features as shown in Fig. 4.

A methodology has been developed that would allow the classification and direction of anisotropy axes in soft magnetic materials to be determined using the in-field measurements of a standard VSM fitted with a relatively simple and inexpensive rotational stage. In this manner the technique reproduces the same simulated torque curves that are associated with more complicated and costly bi-axial VSM instruments.

\section{ACKNOWLEDGMENT}

The authors would like to thank Dr. Serban Lepadatu of the Jeremiah Horrocks Institute for useful discussions. S.B. is supported by a Jeremiah Horrocks Institute studentship. 


\section{REFERENCES}

[1] J. Garcia, J. Carrizo, L. Elbaile, D. Lago-Cachon, M. Rivas, D. Castrillo, and A. Pierna, "Magnetic anisotropy and magnetostriction in nanocrystalline Fe-Al alloys obtained by melt spinning technique," $J$. Magn. Magn. Mat., vol. 372, pp. 27-32, 2014.

[2] V. Dubuget, S. Dubourg, P. Thibaudeau, and F. Duverger, "Magnetic Anisotropy Dispersion With Exchange Energy in Soft Ferromagnetic Thin Films," IEEE Trans. Magn., vol. 46, pp. 1139-1142, 2010.

[3] J. M. Barandiaran, M. Vazquez, A. Hernando, J. Gonzalez, and G. Rivero, "Distribution of the Magnetic-Anisotropy in Amorphous-Alloys Ribbons," IEEE Trans. Magn., vol. 25, pp. 3330-3332, 1989.

[4] Cullity B.D. and Graham C.D., Introduction to Magnetic Materials, 2nd ed John Wiley \& Sons Inc., 2009.

[5] A. Lisfi, S. Pokharel, W. Morgan, G. Warren, and M. Wuttig, "The power of torque magnetometry: defect induced switching in hexaferrite nano-structures," Nanotechnology, vol. 25, article 415702, 2014.

[6] D. Xue, X. Fan, and C. Jiang, "Method for analyzing the in-plane uniaxial anisotropy of soft magnetic thin films," Appl. Phys. Lett., vol 89, article 011910, 2006.

[7] X. Fan, D. Xue, C. Jiang, Y. Gong and J. Li, "An approach for researching uniaxial anisotropy magnet: Rotational magnetization,” $J$.

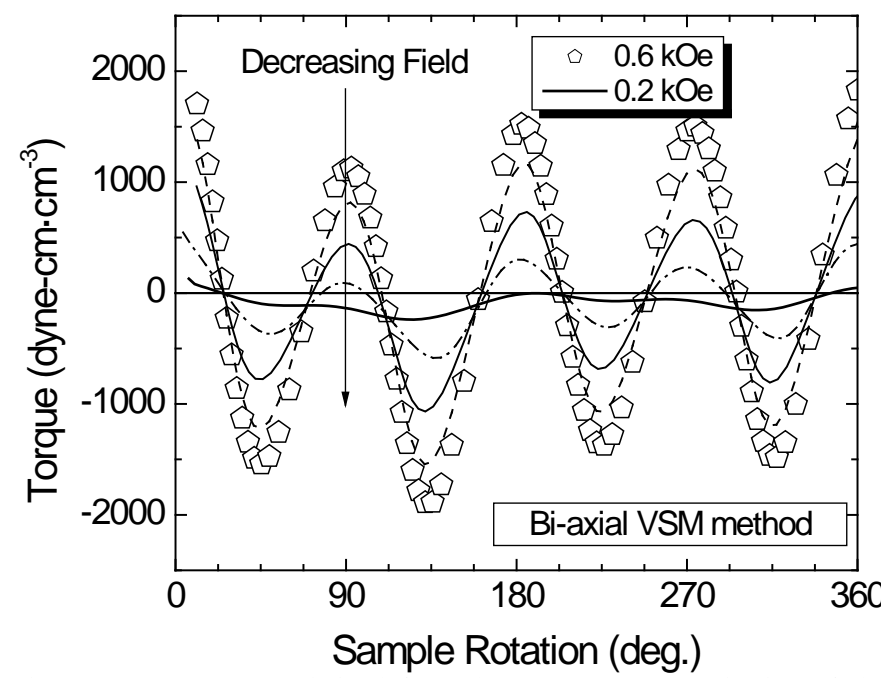

Fig. 1. Torque curves derived from the transverse magnetization of the NFO_100 sample. The sample is initially saturated before the field is reduced to the required value. A measurement of the moment is made every 4 degrees. The intercepts with the abscissa with positive and negative gradients indicate the hard and easy axes of the sample respectively. Each axis is separated from the previous by approximately 45 degrees as expected from the theory.

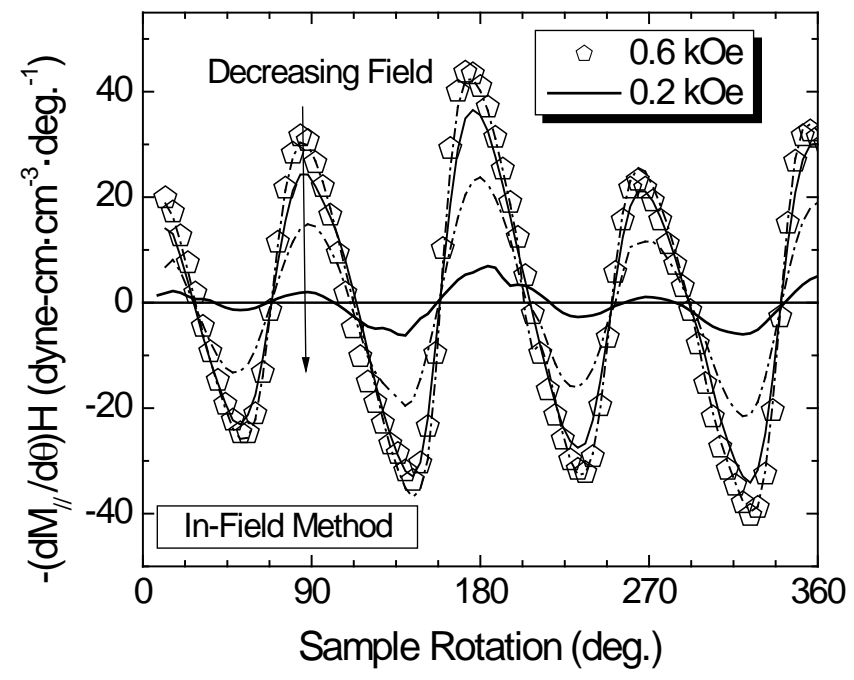

Fig. 2. Torque curves derived from the in-plane magnetization of the NFO_100 showing good agreement with the biaxial method of fig. 1 for the position of the intercepts with the abscissa. This gives confidence in the new method as a means of identifying the hard and easy axes of a material. Note the loss of definition at small fields.
Appl. Phys., vol 102, article 123901, 2007.

[8] M. Vopsaroiu, M. Stewart, T. Fry, M. Cain, and G. Srinivasan, "Tuning the Magneto-Electric Effect of Multiferroic Composites via Crystallographic Texture," IEEE Trans. Magn., vol. 44, pp. 3017-3020, 2008.

[9] A.I.Drokin, N.I.Sudakov, S.Sh.Gendelev, E.M.Kondrat'ev, and L.I.Ryabinkina, "On the Magnetocrystalline Anisotropy of Nickel-Iron Ferrites,” Soviet Physics Journal, vol. 8, pp 109-114, 1965.

[10] K. Yosida and M. Tachiki, "On the Origin of the Magnetic Anisotropy Energy of Ferrites," Progress of Theoretical Physics, vol. 17, pp. 331359, 1957.

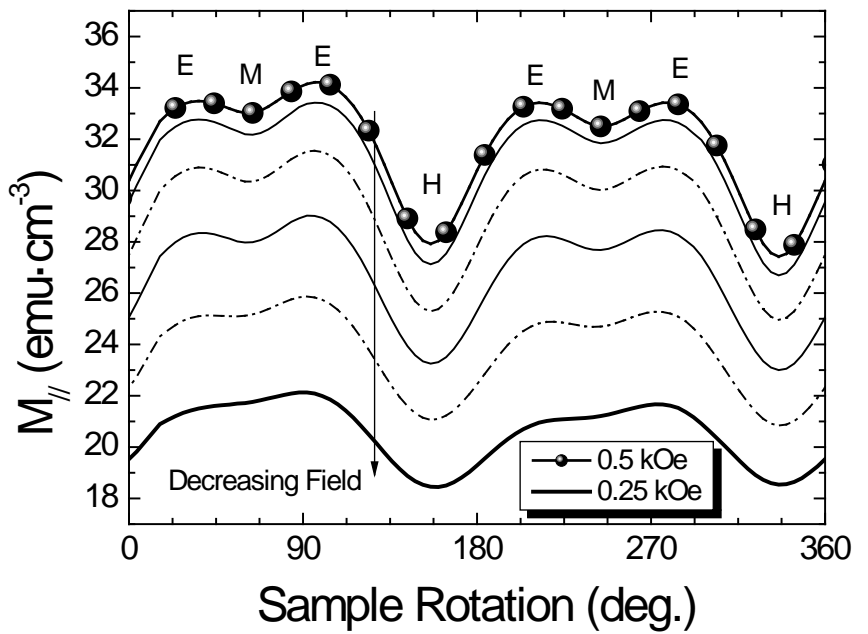

Fig. 3. In-field magnetization as a function of sample rotation for the NFO_110 sample. The sample is initially saturated before the field is reduced to the required value. The turning points are labelled $\mathrm{E}$ for easy axis, $\mathrm{M}$ for medium axis and $\mathrm{H}$ for hard axis.

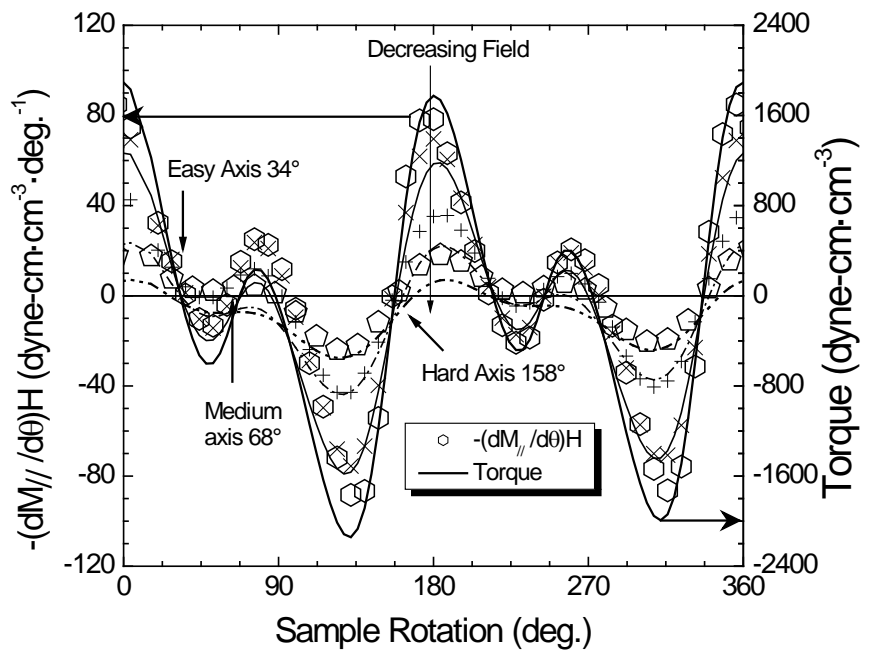

Fig. 4. Comparison of the in-field method (data points) with the bi-axial VSM (lines) for the NFO_110 sample. The good agreement of the intercepts with the abscissa and the relative amplitude of the peaks give confidence in the applicability of the new method 